\title{
Study of DDO 68: nearest candidate for a young galaxy?
}

\author{
S. A. Pustilnik ${ }^{1}$, A. Y. Kniazev ${ }^{2,1}$, and A. G. Pramskij ${ }^{1}$ \\ ${ }^{1}$ Special Astrophysical Observatory RAS, Nizhnij Arkhyz, Karachai-Circassia, 369167 Russia \\ e-mail: sap@sao.ru \\ 2 European Southern Observatory, Garching-bei-Munchen, Germany
}

Received 21 March 2005 / Accepted 8 July 2005

\begin{abstract}
We present the results of optical spectroscopy and imaging with the SAO $6 \mathrm{~m}$ telescope for the dwarf galaxy DDO 68 (UGC 5340 = VV 542), falling into the region of very low density of luminous $\left(L>L_{*}\right)$ galaxies (Lynx-Cancer void). Its deep images in $V, R$ bands and in the narrow $\mathrm{H} \alpha$-filter show that this galaxy has the very irregular morphology, with a long curved tail on the South and a ring-like structure at the Northern edge. The latter consists of 5 separate regions, in three of which we could measure $\mathrm{O} / \mathrm{H}$ by the classical $T_{\mathrm{e}}$ method. Their weighted mean oxygen abundance corresponds to $12+\log (\mathrm{O} / \mathrm{H})=7.21 \pm 0.03$, coincident within uncertainties with those for I Zw 18 . The $(V-R)$ colour of DDO 68 is rather blue all over the galaxy, indicating the youth of its stellar populations. Comparing the $(V-R)^{0}$ colour of the underlying exponential disk of $0.12 \pm 0.04$ with the PEGASE. 2 models for the evolving stellar clusters, we give the first estimate of the ages of the oldest stellar population, which needs confirmation by the other colours and the photometry of resolved stars. These ages are in the range of 200-900 Myr for continuous star formation law, and $\sim 100-115 \mathrm{Myr}$ for the instantaneous starburst. We discuss the properties and the possible youth of this nearby object ( 2.3 times closer than the famous young galaxy I Zw 18) in the context of its atypical environment.
\end{abstract}

Key words. galaxies: photometry - galaxies: evolution - galaxies: abundances - galaxies: interactions galaxies: individual: DDO 68 - cosmology: large scale structure of Universe

\section{Introduction}

Some of dwarf gas-rich galaxies with very low metallicity were considered as probable young galaxies since the seminal paper by Searle \& Sargent (1972). The most metal-poor dwarf I $\mathrm{Zw} 18$ (with the value of $12+\log (\mathrm{O} / \mathrm{H})=7.17-7.21$, e.g., Alloin et al. 1978; Izotov et al. 1999) has for 30 years been the Candidate No. 1 of the local young galaxies. Recently, Izotov $\&$ Thuan (2004) have demonstrated from the very deep Hubble Space Telescope (HST) Colour-Magnitude Diagram (CMD) data that there are no stars in this object older than $0.5 \mathrm{Gyr}$; that is, this nearby object $(D=15 \mathrm{Mpc})$ is indeed a genuine young local galaxy.

There are several other eXtremely Metal-Deficient objects $(\mathrm{XMD}$, conditionally with the characteristic value $Z<$ $1 / 10 Z_{\odot}^{1}$, or $12+\log (\mathrm{O} / \mathrm{H}) \leq 7.65$; e.g., Kunth \& Östlin 2000), considered as good candidates to young local galaxies. First of all, these are the components of the galaxy pair SBS 0335-052 E and $\mathrm{W}$, with $12+\log (\mathrm{O} / \mathrm{H})=7.29$ and 7.12, respectively (e.g., Izotov et al. 1997a; Lipovetsky et al. 1999; Pustilnik et al. 2001, 2004a; Izotov \& Thuan 2005). This unique pair at $D \sim 54 \mathrm{Mpc}$ is, however, 3.6 times more distant than I Zw 18.

1 The solar oxygen abundance is accepted as $12+\log (\mathrm{O} / \mathrm{H})=8.66$ according to Asplund et al. (2004).
In our discussion, we distinguish truly young galaxies (similar to I $\mathrm{Zw} \mathrm{18),} \mathrm{in} \mathrm{which} \mathrm{their} \mathrm{young} \mathrm{stellar} \mathrm{generation} \mathrm{is}$ made up of pregalactic matter, from the so-called tidal young dwarfs (e.g., Duc \& Mirabel 1999), similar to Holmberg IX in the vicinity of M 81. Holmberg IX is devoid of old Red Giant Branch (RGB) stars (Makarova et al. 2002), so its star formation began recently. But its gas metal content is close to that of the massive parent galaxy ISM, from which it was recently formed, so in this context such tidal dwarfs should be treated not as young but as rejuvenated.

It is worth noting that the very low ISM metallicity is considered only as an indication of the possible unevolved status of a galaxy. In particular, from the theoretical point of view, several evolutionary scenarios can lead to such a result. Briefly, they include: a) the significant metal loss due to galactic superwinds in the shallow gravitational wells of dwarf galaxies; b) the inflow and the mixture of an unevolved intergalactic gas to an evolved galaxy; c) the very slow astration and related ISM enrichment characteristic of the very low surface brightness (LSB) galaxies; and d) the recent onset of the first star formation episode. All but one correspond to an aged galaxy. Moreover, observationally among 16 known XMD galaxies at distances less than $15 \mathrm{Mpc}$, at least nine objects, either from the CMD analysis (e.g., Sextans A and B, GR 8, Leo A) or from the red colours of the unresolved stellar population in their outer regions (e.g., UGCA 20, UGC 2684, 

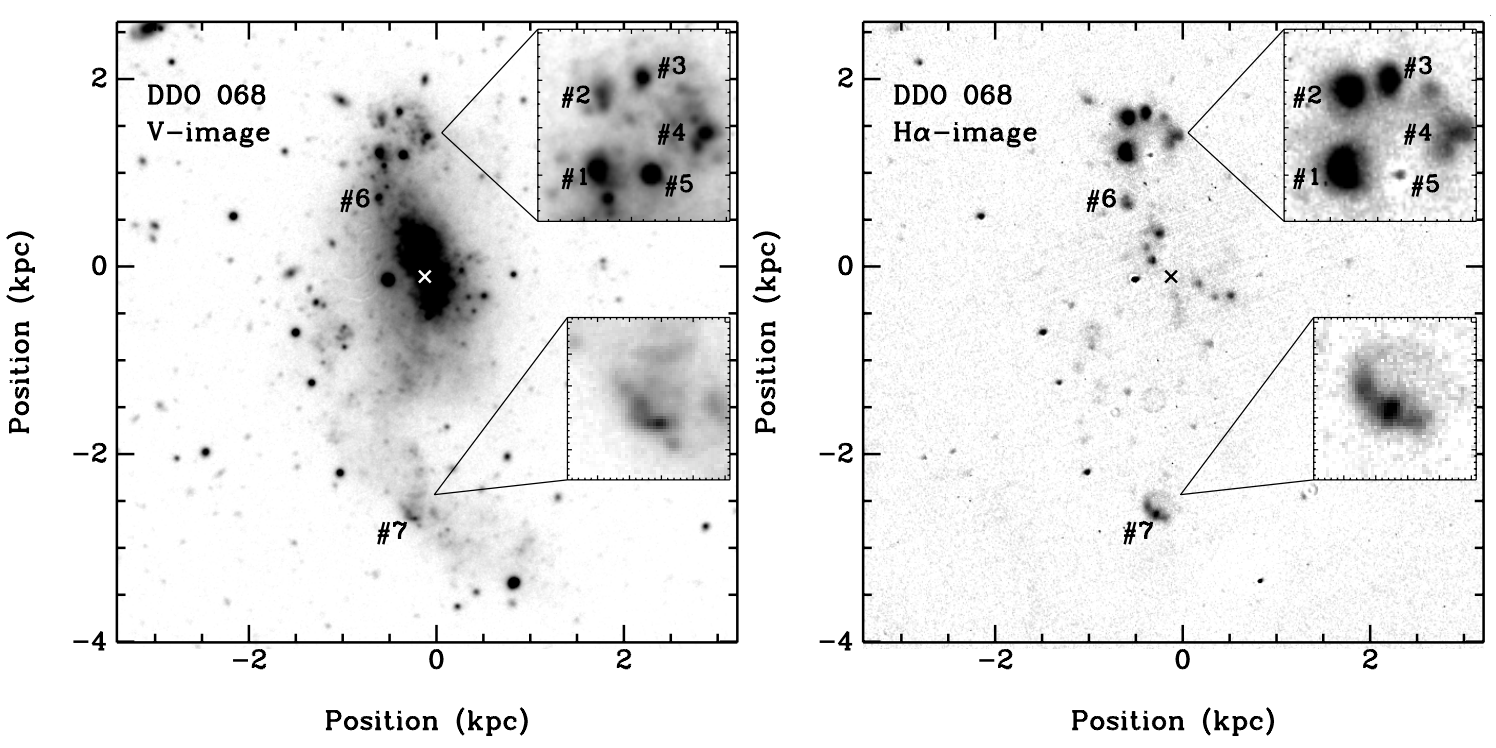

Fig. 1. The $V$-band and net $\mathrm{H} \alpha$ images of DDO 68 showing its overall morphology and the regions of the currently intense star formation. The numbers mark the bright knots whose spectra are discussed in the paper. The cross marks the central position for building the surface brightness profiles in Fig. 3. The scale is 31.5 pc per 1". The insets show blow-ups of the ring-like features.

van Zee et al. 1996, 1997), are recognized as old systems. Only one, I Zw 18, is now considered as a truly young object.

In searches for new XMD galaxies, we found that some of them populate the "void" regions, where the distances from the void centers to luminous galaxies ( $L>L_{*}, L_{*}$ correspond to $M_{B}=-19.6$ for $H=72 \mathrm{~km} \mathrm{~s}^{-1} \mathrm{Mpc}^{-1}$, assumed in the paper) exceed 4-5 Mpc for the smallest voids and 8-10 Mpc for the more typical voids. In particular, the XMD blue compact dwarf (BCD) galaxy HS $0822+3542$ with $12+\log (\mathrm{O} / \mathrm{H})=7.38$, appeared near the centre of the nearby small Lynx-Cancer void (Kniazev et al. 2000; Pustilnik et al. 2003b). Voids delineated by luminous, massive galaxies are not absolutely empty. Some numbers of the lower-mass galaxies fill in voids (e.g., Salzer 1989; Pustilnik et al. 1995; Lindner et al. 1996; Popescu et al. 1997; Grogin \& Geller 2000, among others), probably forming the substructures of the filament types (Lindner et al. 1996; Gottlöber et al. 2003; Fairall et al. 2004). The very low-density environment in voids and the scaled-down mass spectrum of the preformed DM halos, coupled with the effect of reionization, are all expected to cause formation of a somewhat different galaxy population from any in more typical, denser environments (e.g., Peebles 2001; Gottlöber et al. 2003). A significantly reduced probability of galaxy interactions in voids, which is suggested as an important factor of star formation history (e.g., Rojas et al. 2004), can cause the differences in the rate of chemical enrichment.

Some indication of the less evolved status of low-mass galaxies in voids was presented, e.g., by Huchtmeier et al. (1997) and Pustilnik et al. (2002), based on H I data. The higher star formation (SF) activity of void galaxies in respect to "wall" galaxies, detected by Grogin \& Geller (2000) and Rojas et al. (2004), also indicates their larger available gas fuel reservoir. However, the metallicity issue of the void galaxy population has not yet been properly addressed, mainly due to observational difficulties. Only the study of absorption lines in $\operatorname{Ly} \alpha$ clouds with low column densities in various environments led to the conclusion that such objects in voids show a significantly lower content of heavy elements (Lu et al. 1998).

Since the Lynx-Cancer void is one of the nearest $\left(D_{\text {center }} \sim\right.$ $11 \mathrm{Mpc}$ ), many of the underluminous galaxies falling in this region are sufficiently bright, so that in contrast to the situation in more distant voids, even inconspicuous H II regions in more or less typical late-type dwarfs are available for determining chemical abundance in "routine" programs. Therefore, it was tempting to search in this void for some other XMD objects, including likely young galaxy candidates. To that end we performed the spectrophotometry of ten dwarfs in this region, and the data will be presented separately elsewhere. In this paper we report the discovery of an extremely low oxygen abundance in $\mathrm{dIm} / \mathrm{BCD}$ galaxy DDO 68 (UGC $5340=$ VV 542, J2000 coordinates $09: 56: 45.7+28: 49: 35)$, situated at the periphery of this void at a distance of $D \sim 6.5 \mathrm{Mpc}$. We also present the results of $V, R$, and $\mathrm{H} \alpha$ imaging, indicate its very blue colours, and discuss DDO 68 properties that make it a probable candidate for a young galaxy.

\section{Observations and reduction}

All observations were conducted with the SCORPIO multimode instrument (Afanasiev \& Moiseev 2005) installed in the prime focus of the SAO $6 \mathrm{~m}$ telescope (BTA), during 2 runs - in November 9-11, 2004 and January 7-13, 2005. For the longslit spectral observations the grism VPH550g was used with the $2 \mathrm{~K} \times 2 \mathrm{~K} \mathrm{CCD}$ detector EEV 42-40, with the exposed region of $2048 \times 600$ px. This gave the range 3500-7500 $\AA$ with $\sim 2.0 \AA$ pixel $^{-1}$ and $F W H M \sim 12 \AA$ along the dispersion. The scale along the slit was $0{ }^{\prime} 18$ pixel $^{-1}$ and total extent of $\sim 2^{\prime}$. The spectra were obtained for several slit positions, crossing the bright knots, marked by their numbers on the $V$-band image of the galaxy obtained also in this program (Fig. 1). Exposure 
Table 1. Journal of the $6 \mathrm{~m}$ telescope observations of DDO 68 .

\begin{tabular}{ccccccc}
\hline \hline Date & $\begin{array}{c}\text { Exposure } \\
\text { time }[\mathrm{s}] \\
(2)\end{array}$ & $\begin{array}{c}\text { Wavelen. range }[\AA] \\
\text { or Filter } \\
(3)\end{array}$ & $\begin{array}{c}\text { Dispersion } \\
{[\AA / \text { pixel] }} \\
(4)\end{array}$ & $\begin{array}{c}\text { Seeing } \\
{[\operatorname{arcsec}]} \\
(5)\end{array}$ & Airmass & $\begin{array}{c}\text { PA } \\
{[\text { degree }]} \\
(7)\end{array}$ \\
\hline 09.11 .2004 & $2 \times 900$ & $3500-7500$ & 2.0 & 0.8 & 1.15 & 90 \\
09.11 .2004 & $1 \times 900$ & $3500-7500$ & 2.0 & 0.8 & 1.08 & 123 \\
08.01 .2005 & $4 \times 900$ & $3500-7500$ & 2.0 & 1.7 & 1.07 & 0 \\
13.01 .2005 & $3 \times 900$ & $3500-7500$ & 2.0 & 1.4 & 1.09 & 123 \\
12.01 .2005 & $3 \times 600$ & $V$ & - & 1.8 & 1.07 & - \\
12.01 .2005 & $3 \times 600$ & $R$ & - & 1.7 & 1.20 & - \\
12.01 .2005 & $1 \times 900$ & SED665 & - & 1.7 & 1.27 & - \\
12.01 .2005 & $1 \times 900$ & SED607 & - & 1.7 & 1.33 & - \\
\hline
\end{tabular}

times varied between 15 to $60 \mathrm{~min}$. When longer than $15 \mathrm{~min}$, they were broken into subexposures of 15-min duration. The objects spectra were complemented before or after by the reference spectra of He-Ne-Ar lamp for the wavelength calibration. Bias and flat-field images were also acquired to perform the standard reduction of 2D spectra. Spectral standard star Feige 34 (Bohlin 1996) was observed during the night for the flux calibration.

The primary task of these observations was to get high S-to-N spectra of several H II regions in order to detect the faint line [O III] $\lambda 4363$ and to directly measure their electron temperatures $T_{\mathrm{e}}$ and oxygen abundances. Seeing was $\sim 00^{\prime} 8$ in the November 2004 run and $\sim 1$ '. $4-1$ ".7 in the January 2005 run. We also used SCORPIO in the imaging mode $(2048 \times$ 2048 px, binned $2 \times 2$, the field of view of $\sim 6^{\prime} \times 6^{\prime}$ ) to study DDO 68 morphology, structure and colours. The standard Johnson-Cousins $V, R_{\mathrm{c}}$ and the middle-band filters for $\mathrm{H} \alpha$ line images SED665 (central wavelength $=6622 \AA, F W H M=$ $191 \AA$ ) and SED607 (central wavelength $=6063 \AA, F W H M=$ $167 \AA$ ) were used. On the night of January 12, 2005, we obtained 30-min exposures of the galaxy in $V$ and $R$ bands (all broken into 10-min subexposures), and 15-min exposures in both SED665 and SED607 filters. The night was photometric, with the seeing between 1 '. 6 and 1". 8 . Bias and flat-field images were acquired to perform the standard reduction. For the broad-band calibration, we observed the standard stars during the night in the field of QSO FBQ 0951+263 (Nakos et al. 2003), while for the calibration of $\mathrm{H} \alpha$ images we observed the spectrophotometric standard Feige 34. The coefficients of the resulting photometric system have the overall uncertainties of $0 \mathrm{~m} 046$ in $V$ and $0 \mathrm{~m} 056$ in $R$. The standard pipeline with the use of IRAF $^{2}$ and MIDAS ${ }^{3}$ was applied for the reduction of both long-slit spectra and images, which included the next steps.

Cosmic ray hits were removed from all spectra and direct images in MIDAS. Using IRAF packages from CCDRED for spectra and images, we subtracted bias, and performed flatfield correction. After that, spectra were wavelength-calibrated.

${ }^{2}$ IRAF: the Image Reduction and Analysis Facility is distributed by the National Optical Astronomy Observatory, which is operated by the Association of Universities for Research in Astronomy (AURA) under cooperative agreement with the National Science Foundation (NSF).

3 MIDAS is an acronym for the European Southern Observatory package - Munich Image Data Analysis System.
Night sky background was subtracted from all spectra. Then, using the data on spectrophotometry standard stars, all spectra were transformed to absolute fluxes. One-dimensional spectra were extracted by summing up, without weighting, various numbers of rows along the slit depending on exact region of interest. The next step of the reduction of direct images consisted of shifting all $V, R, S E D 607$, and SED665 images into the common world coordinate system. Finally, all subexposures were combined into one image by summation.

To obtain the integrated photometry, the $V$ and $R$ surface brightness profiles (SBPs) and the fitting of SBPs, the method and the programs described in detail in Kniazev et al. (2004a) were used: (1) $V$ and $R$ images were combined and filtered with a smooth-and-clip filter (Shergin et al. 1996); (2) the galaxy was detected above the $3 \sigma$ noise level on the combined and filtered image and the mask-frame was created to show the location of the galaxy for the subsequent steps of the analysis; (3) all background galaxies and stars were additionally masked; (4) using the mask-frame, the integrated photometry was calculated, the SBPs were created for each filter with the circular apertures with the step of $2^{\prime \prime}$, and the errors were calculated; (5) fitting of SBPs by the exponential disc was performed with weights $w_{k}=\sigma_{k}^{-1}$, where $\sigma_{k}$ is the error of the flux calculated within the circular aperture for each SB level in the previous module.

In the three $\mathrm{H}$ II regions (Knots 1, 2, and 3 in the Northern ring/oval) where the line [O III] $\lambda 4363$ was seen, we extracted only the regions along the slit where this line was well above the noise (from 1". 8 to 3 ". 8 for various knots). All emission lines were measured by applying the MIDAS programs described in detail in a recent paper by Kniazev et al. (2004b). Briefly, they draw continuum, perform robust noise estimation, fit separate lines by a single Gaussian superimposed on the continuumsubtracted spectrum and integrate the line flux. The emission lines, blended in pairs or triplets, were fitted simultaneously as blend of two or more Gaussian features. The quoted errors of singular line intensities include the following components. The first is related to the Poisson statistics of the line photon flux. The second component is the error resulting from the creation of the underlying continuum, which gives the main contribution to the errors of faint lines. For the fluxes of the lines in blends, an additional error appears related to the goodness of fit. Last, the term related to the uncertainty of the spectral 
sensitivity curve gives an additional error to the relative line intensities. This term is $5 \%$ for the observations presented, hence, it gives the main contribution to errors of the relative intensities of strong lines. All these components are summed squared, and the total errors have been propagated to calculate the errors of all derived parameters.

\section{Results}

\subsection{Line intensities and element abundances}

The relative intensities of all emission lines used for abundance determination in the 3 discussed H II regions (Knots 1 , 2 , and 3), as well as the derived $\mathrm{CH} \beta, E W$ s of Balmer absorption lines, measured flux in $\mathrm{H} \beta$ emission line, and the measured heliocentric radial velocities of each knot, are given in Tables 2 and 3. The position angle (PA) of the slit and the seeing $\theta$ during observation are also given for all spectra. The best $1 \mathrm{D}$ spectra for each of these regions, as well as the spectra of Knots 5 and 7, are shown in Fig. 2. For each of Knots 1, 2, and 3, we got repeated observations and can compare individual data. For Knot 1 the relative line intensities are very consistent in the two spectra for the most of lines except [O II] $\lambda 3727$ and $\mathrm{H} \alpha$. This presumably is due to the different slit position on the asymmetric $\mathrm{H}$ II region and also due to a factor of two different seeings, resulting in some contribution from the outer parts of this knot. For Knot 2 the first two spectra are very consistent with each other, while the 3-d indicates some additional "reddening". The similar situation is for the 2 nd spectrum of Knot $3\left(\mathrm{PA}=-57^{\circ}\right.$, $\theta=1$." 4 ), extracted from the same $2 \mathrm{D}$ spectrum. This seems to indicate some additional error in the spectral sensitivity curve for this night. However, the effect of this on the resulting abundances is small.

Extinction in the observed $\mathrm{H}$ II regions is low: $\mathrm{C}(\mathrm{H} \beta) \lesssim 0.1$. A larger value of $\mathrm{C}(\mathrm{H} \beta)$, derived for one spectrum of Knots 2 and $3(\sim 0.2-0.3)$, is probably due to the worse quality of the spectral sensitivity curve for this night. This low $\mathrm{C}(\mathrm{H} \beta)$ is quite consistent with the extinction data for most very metal-poor galaxies.

Chemical abundances and physical parameters are determined in the frame of the classical two-zone model of H II region (Stasińska 1990), as described, e.g., in our recent papers by Pustilnik et al. (2004b) and by Kniazev et al. (2004b), which in turn follow the method described by Izotov et al. (1997b). The derived individual abundances of oxygen for Knots 1, 2, and 3, as well as their $T_{\mathrm{e}}$, are given in Table 4. Since the [S II] $\lambda 6717 / \lambda 6731$ line ratio is higher than 1.4 for all spectra, the density $N_{\mathrm{e}}=10 \mathrm{~cm}^{-3}$ was accepted in all our calculations (Aller 1984).

Since the line fluxes from these knots are rather small, the S-to-N in the principal line [O III] $\lambda 4363$ was at most $\sim 8$. Therefore the majority of resulting abundances for individual observations are of medium accuracy. However, they all are consistent each to the other within their cited uncertainties. Thus, we average them and accept the mean values for each of the three regions: $12+\log (\mathrm{O} / \mathrm{H})=7.23 \pm 0.05,7.21 \pm 0.05$, and $7.03 \pm 0.08$, for Knots 1,2 , and 3, respectively. These mean values for different knots are consistent each to the other

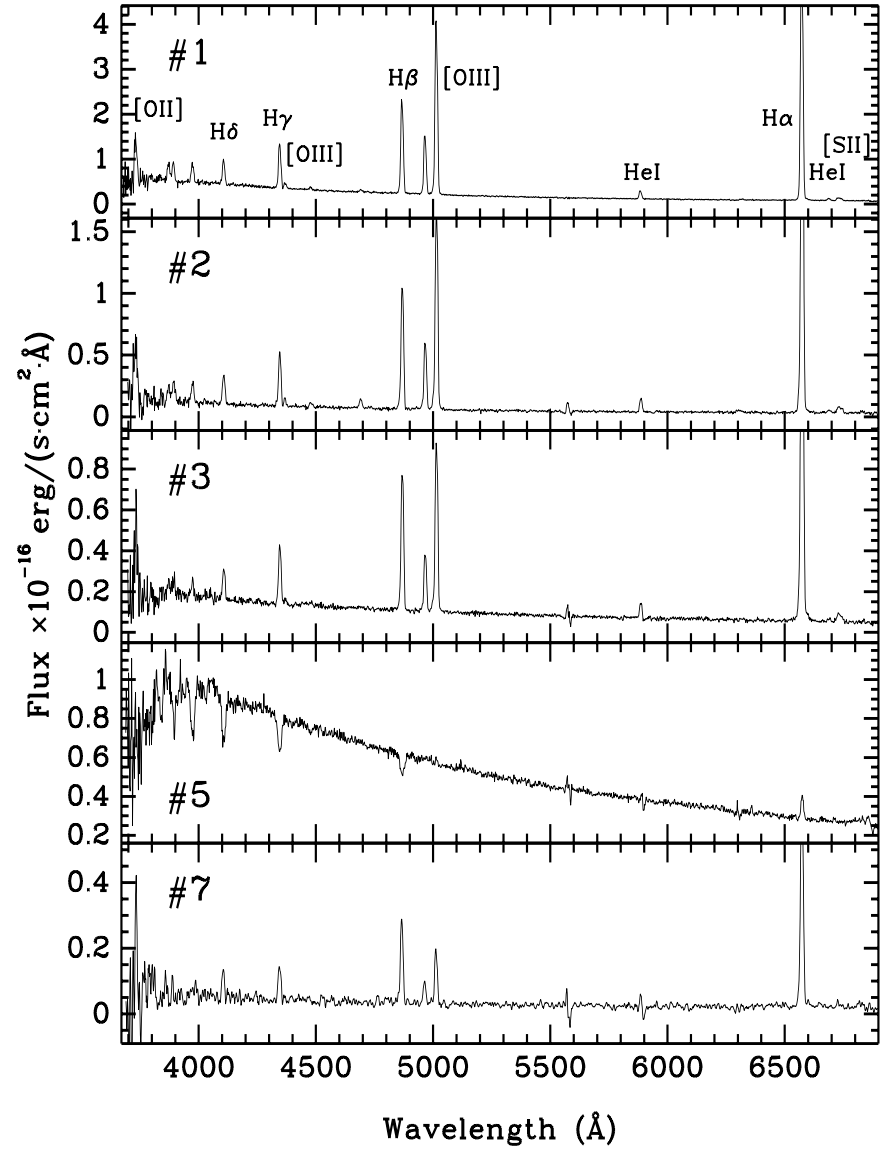

Fig. 2. The spectra of 5 knots in DDO 68 (see nomenclature in Fig. 1). Main lines are marked on the spectrum of Knot 1 . The spectrum of Knot 7 (with no reliable $\mathrm{O} / \mathrm{H}$ ) is presented to show the general similarity to those of other knots that also indicate low $\mathrm{O} / \mathrm{H}$.

within their uncertainties, so we take their mean as a measure of the heavy element abundances of DDO 68 (at least, in this northern "ring"). If all seven measurements of $\mathrm{O} / \mathrm{H}$ in these 3 knots are considered as the independent representatives of a unique value for all three knots, their weighted mean corresponds to $12+\log (\mathrm{O} / \mathrm{H})=7.17 \pm 0.03$. The 1 st measurement for Knot 3, with $12+\log (\mathrm{O} / \mathrm{H})=6.95 \pm 0.14$, looks, however, a bit outlying. If we exclude this from the general mean, then the resulting mean value of the remaining six measurements (which probably is more conservative) corresponds to $12+\log (\mathrm{O} / \mathrm{H})=7.21 \pm 0.03$. We take this $\mathrm{O} / \mathrm{H}$ as a characteristic value of DDO 68 in further discussion.

For Knot 1 the estimates of $\mathrm{Ne}, \mathrm{S}$, and $\mathrm{Ar}$ abundances were also derived. They correspond to $\log (\mathrm{Ne} / \mathrm{O})=-0.70 \pm 0.10$, $\log (\mathrm{S} / \mathrm{O})=-1.21 \pm 0.16$, and $\log (\mathrm{Ar} / \mathrm{O})=-2.20 \pm 0.07$. Within rather large uncertainties, they are consistent with the abundance ratios for the most metal-deficient blue compact galaxies (BCGs) presented by Izotov \& Thuan (1999).

Having the $E W \mathrm{~s}$ of emission line $\mathrm{H} \beta$, we can estimate the ages of starbursts in these three H II regions apparently placed along a more or less regular oval. The average values of $E W(\mathrm{H} \beta)$ for Knots 1-3 are given in Table 5. For Knots 4, 6, and 7, we got spectra of relatively low S-to-N, so we do not discuss them in detail. However, their $E W \mathrm{~s}(\mathrm{H} \beta)$ are well measured 
Table 2. Line intensities in Knot 1 of DDO 68.

\begin{tabular}{|c|c|c|c|c|}
\hline \multirow[b]{2}{*}{$\lambda_{0}(\AA)$ Ion } & \multicolumn{2}{|c|}{$\mathrm{PA}=90^{\circ}, \theta=0 \prime^{\prime} 8$} & \multicolumn{2}{|c|}{$\mathrm{PA}=0^{\circ}, \theta=1^{\prime \prime} .6$} \\
\hline & $F(\lambda) / F(\mathrm{H} \beta)$ & $I(\lambda) / I(\mathrm{H} \beta)$ & $F(\lambda) / F(\mathrm{H} \beta)$ & $I(\lambda) / I(\mathrm{H} \beta)$ \\
\hline 3727 [O II] & $0.196 \pm 0.009$ & $0.190 \pm 0.011$ & $0.552 \pm 0.028$ & $0.545 \pm 0.030$ \\
\hline 3869 [Ne III] & $\ldots$ & $\ldots$ & $0.184 \pm 0.016$ & $0.182 \pm 0.017$ \\
\hline $3967[\mathrm{Ne}$ III $]+\mathrm{H} 7$ & $0.188 \pm 0.011$ & $0.253 \pm 0.019$ & $0.215 \pm 0.011$ & $0.235 \pm 0.019$ \\
\hline $4101 \mathrm{H} \delta$ & $0.201 \pm 0.011$ & $0.266 \pm 0.019$ & $0.242 \pm 0.013$ & $0.260 \pm 0.019$ \\
\hline $4340 \mathrm{H} \gamma$ & $0.423 \pm 0.023$ & $0.473 \pm 0.029$ & $0.466 \pm 0.024$ & $0.477 \pm 0.027$ \\
\hline 4363 [O III] & $0.047 \pm 0.07$ & $0.046 \pm 0.007$ & $0.058 \pm 0.007$ & $0.057 \pm 0.007$ \\
\hline 4686 He II & $0.022 \pm 0.003$ & $0.022 \pm 0.003$ & $0.033 \pm 0.004$ & $0.033 \pm 0.004$ \\
\hline $4861 \mathrm{H} \beta$ & $1.000 \pm 0.005$ & $1.000 \pm 0.006$ & $1.000 \pm 0.004$ & $1.000 \pm 0.005$ \\
\hline 4959 [O III] & $0.646 \pm 0.033$ & $0.616 \pm 0.033$ & $0.624 \pm 0.032$ & $0.616 \pm 0.032$ \\
\hline 5007 [O III] & $1.933 \pm 0.097$ & $1.838 \pm 0.096$ & $1.899 \pm 0.095$ & $1.876 \pm 0.095$ \\
\hline $5876 \mathrm{He} \mathrm{I}$ & $0.102 \pm 0.007$ & $0.093 \pm 0.006$ & $0.079 \pm 0.006$ & $0.078 \pm 0.006$ \\
\hline $6300[\mathrm{O} \mathrm{I}]$ & $0.021 \pm 0.003$ & $0.019 \pm 0.003$ & $\ldots$ & $\ldots$ \\
\hline 6312 [S III] & $0.010 \pm 0.003$ & $0.009 \pm 0.003$ & $\ldots$ & $\ldots$ \\
\hline $6563 \mathrm{H} \alpha$ & $3.102 \pm 0.159$ & $2.772 \pm 0.161$ & $2.740 \pm 0.139$ & $2.711 \pm 0.151$ \\
\hline 6584 [N II] & & $\ldots$ & $0.017 \pm 0.034$ & $0.016 \pm 0.034$ \\
\hline $6678 \mathrm{He} \mathrm{I}$ & $0.024 \pm 0.004$ & $0.021 \pm 0.003$ & $0.024 \pm 0.004$ & $0.024 \pm 0.004$ \\
\hline 6717 [S II] & $0.035 \pm 0.006$ & $0.031 \pm 0.005$ & $0.034 \pm 0.005$ & $0.033 \pm 0.005$ \\
\hline $6731[\mathrm{~S} \mathrm{II}]$ & $0.021 \pm 0.006$ & $0.019 \pm 0.005$ & $0.018 \pm 0.004$ & $0.018 \pm 0.004$ \\
\hline 7136 [Ar III] & $0.026 \pm 0.004$ & $0.023 \pm 0.004$ & $0.019 \pm 0.003$ & $0.019 \pm 0.008$ \\
\hline $\mathrm{C}(\mathrm{H} \beta) \operatorname{dex}$ & \multicolumn{2}{|c|}{$0.10 \pm 0.07$} & \multicolumn{2}{|c|}{$0.00 \pm 0.07$} \\
\hline$E W($ abs $) \AA$ & \multicolumn{2}{|c|}{$5.05 \pm 0.82$} & \multicolumn{2}{|c|}{$1.35 \pm 0.75$} \\
\hline$F(\mathrm{H} \beta)^{a}$ & \multicolumn{2}{|c|}{$24.8 \pm 1.3$} & \multicolumn{2}{|c|}{$27.2 \pm 1.4$} \\
\hline$E W(\mathrm{H} \beta) \AA$ & \multicolumn{2}{|c|}{$117 \pm 0.6$} & \multicolumn{2}{|c|}{$110 \pm 0.5$} \\
\hline Rad. vel. $\mathrm{km} \mathrm{s}^{-1}$ & \multicolumn{2}{|c|}{$450 \pm 48$} & \multicolumn{2}{|c|}{$378 \pm 33$} \\
\hline
\end{tabular}

${ }^{a}$ In units of $10^{-16} \mathrm{ergs} \mathrm{s}^{-1} \mathrm{~cm}^{-2}$.

and can also be used to date the starbursts at their respective locations.

We use the latest version (v5) of the models from Starburst99 (Leitherer et al. 1999; Vazquez \& Leitherer 2005) with the lowest metallicity given their $(z=0.001)$ to estimate ages of instantaneous starbursts from the observed $E W \mathrm{~s}(\mathrm{H} \beta)$. For the Salpeter IMF with $M_{\text {low }}$ and $M_{\text {up }}$ of $0.1 M_{\odot}$ and $100 M_{\odot}$, respectively, the derived ages of all emission knots are also given in Table 5. They range from 3 to 7 Myr. An independent check of the ages of all knots can be performed using the $E W(\mathrm{H} \alpha)$ from the obtained $\mathrm{H} \alpha$ images. Both age estimates are very consistent to each other.

Knot 5 (see Fig. 1) appeared to be a compact young stellar cluster with well-seen Balmer series in absorption, besides $\mathrm{H} \alpha$, on which some emission is superimposed. The radial velocity of this cluster derived on these lines $\left(V_{\text {hel }}=396 \pm 90 \mathrm{~km} \mathrm{~s}^{-1}\right)$ is quite consistent with those for the H II regions in the "ring" and with the systemic velocity of DDO 68. The measured $E W \mathrm{~s}$ of absorption lines $\mathrm{H} \beta, \mathrm{H} \gamma, \mathrm{H} \delta$ are in the range of 4.4-5.8 (see Table 6). The value of $E W$ (emis. $\mathrm{H} \alpha$ ) after correction for the underlying absorption is $9.5 \pm 0.5 \AA$. The $E W$ of absorption $\mathrm{H} \beta$, in turn, after the correction for emission component, is $5.8 \pm 0.5 \AA$. $E W$ s of $\mathrm{H}_{8}$ and $\mathrm{H}_{9}$ are measured with large uncertainties and are not taken for comparison. We compared all $E W$ s of Balmer absorptions with the respective model values, presented by Gonzalez Delgado et al. (1999) for the cluster with metallicity $z=0.001$ (nearest to the observed one for DDO 68), assuming the instantaneous starburst with the
Salpeter IMF with $M_{\text {low }}$ and $M_{\text {up }}$ of 1 and $80 M_{\odot}$. The $E W \mathrm{~s}$ of emission $\mathrm{H} \alpha$ and the extrapolated one of $\mathrm{H} \beta$ were compared to predictions of the latest version of Starburst99 (Vazquez \& Leitherer 2005) for the Salpeter IMF with $M_{\text {up }}=100 M_{\odot}$. Both absorption and emission line $E W$ values are consistent with the cluster age of $T_{\text {cluster }} \sim 22-23 \mathrm{Myr}$.

Thus, we conclude that starbursts in 4 sites along the "ring" are synchronized within the interval of 2 Myr. Only in Knot 5 did the star formation episode begin $\sim 18$ Myr earlier than in the rest positions. One of the possible options for producing such a configuration of starbursts is the induced gas collapse behind the front of shock wave, generated by the previous strong starburst (e.g., van Dyk et al. 1998; Efremov et al. 2002; Elmegreen et al. 2004). However, the outlying age of Knot 5 suggests more complicated SF scenario in this region.

\subsection{Results of imaging}

The best quality image of DDO 68 obtained in these observations is the one in $V$-band. This is shown in Fig. 1 with the surface brightness cuts allowing the maximal extent of the galaxy to be followed. Also on this image we mark the knots for which the spectra, discussed in the previous section, were acquired. The net $\mathrm{H} \alpha$ image in Fig. 1 displays the regions of current SF. We emphasise that besides the Northern "ring" with diameter of $\sim 500 \mathrm{pc}$ and rather bright emission-line knots along this "ring", there is a similar structure in the Southern part of the galaxy that is connected with the Southern tail: Knot 7 in 
Table 3. Line intensities in Knots 2, 3 and 7 of DDO 68.

\begin{tabular}{|c|c|c|c|c|c|c|}
\hline \multirow[b]{2}{*}{$\lambda_{0}(\AA)$ Ion } & \multicolumn{2}{|c|}{ Knot $2, \mathrm{PA}=-57^{\circ}, \theta=0 . .^{\prime} 8$} & \multicolumn{2}{|c|}{ Knot $2, \mathrm{PA}=0^{\circ}, \theta=1 . .6$} & \multicolumn{2}{|c|}{ Knot $2, \mathrm{PA}=-57^{\circ}, \theta=1^{\prime \prime} .4$} \\
\hline & $F(\lambda) / F(\mathrm{H} \beta)$ & $I(\lambda) / I(\mathrm{H} \beta)$ & $F(\lambda) / F(\mathrm{H} \beta)$ & $I(\lambda) / I(\mathrm{H} \beta)$ & $F(\lambda) / F(\mathrm{H} \beta)$ & $I(\lambda) / I(\mathrm{H} \beta)$ \\
\hline 3727 [O II] & $0.464 \pm 0.025$ & $0.467 \pm 0.027$ & $0.451 \pm 0.023$ & $0.451 \pm 0.025$ & $0.698 \pm 0.035$ & $0.798 \pm 0.045$ \\
\hline $3967[\mathrm{Ne}$ III $]+\mathrm{H} 7$ & $\ldots$ & $\ldots$ & $0.221 \pm 0.013$ & $0.221 \pm 0.019$ & $0.176 \pm 0.011$ & $0.227 \pm 0.021$ \\
\hline $4101 \mathrm{H} \delta$ & $0.247 \pm 0.022$ & $0.263 \pm 0.023$ & $0.264 \pm 0.015$ & $0.264 \pm 0.020$ & $0.230 \pm 0.013$ & $0.280 \pm 0.022$ \\
\hline $4340 \mathrm{H} \gamma$ & $0.470 \pm 0.033$ & $0.474 \pm 0.046$ & $0.486 \pm 0.026$ & $0.486 \pm 0.028$ & $0.424 \pm 0.024$ & $0.469 \pm 0.029$ \\
\hline $4363[\mathrm{O}$ III] & $0.049 \pm 0.018$ & $0.049 \pm 0.018$ & $0.049 \pm 0.008$ & $0.049 \pm 0.008$ & $0.051 \pm 0.008$ & $0.053 \pm 0.009$ \\
\hline 4686 He II & $0.121 \pm 0.015$ & $0.120 \pm 0.015$ & $0.096 \pm 0.007$ & $0.096 \pm 0.007$ & $0.082 \pm 0.008$ & $0.082 \pm 0.009$ \\
\hline $4861 \mathrm{H} \beta$ & $1.000 \pm 0.017$ & $1.000 \pm 0.022$ & $1.000 \pm 0.007$ & $1.000 \pm 0.007$ & $1.000 \pm 0.007$ & $1.000 \pm 0.008$ \\
\hline 4959 [O III] & $0.517 \pm 0.031$ & $0.515 \pm 0.031$ & $0.524 \pm 0.027$ & $0.524 \pm 0.027$ & $0.537 \pm 0.028$ & $0.522 \pm 0.028$ \\
\hline 5007 [O III] & $1.538 \pm 0.078$ & $1.532 \pm 0.078$ & $1.548 \pm 0.077$ & $1.548 \pm 0.078$ & $1.620 \pm 0.081$ & $1.564 \pm 0.080$ \\
\hline $5876 \mathrm{He} \mathrm{I}$ & $0.087 \pm 0.012$ & $0.086 \pm 0.012$ & $0.061 \pm 0.005$ & $0.061 \pm 0.005$ & $0.087 \pm 0.007$ & $0.077 \pm 0.006$ \\
\hline 6300 [O I] & $\ldots$ & $\ldots$ & $\ldots$ & $\ldots$ & $0.020 \pm 0.010$ & $0.017 \pm 0.009$ \\
\hline 6312 [S III] & $\ldots$ & $\ldots$ & $\ldots$ & $\ldots$ & $0.015 \pm 0.012$ & $0.013 \pm 0.011$ \\
\hline $6563 \mathrm{H} \alpha$ & $2.777 \pm 0.140$ & $2.740 \pm 0.151$ & $2.651 \pm 0.133$ & $2.651 \pm 0.144$ & $3.286 \pm 0.167$ & $2.744 \pm 0.155$ \\
\hline $6678 \mathrm{He} \mathrm{I}$ & $0.034 \pm 0.015$ & $0.030 \pm 0.015$ & $0.012 \pm 0.004$ & $0.012 \pm 0.004$ & $0.028 \pm 0.007$ & $0.023 \pm 0.006$ \\
\hline 6717 [S II] & $0.066 \pm 0.017$ & $0.065 \pm 0.017$ & $0.035 \pm 0.007$ & $0.035 \pm 0.007$ & $0.056 \pm 0.009$ & $0.046 \pm 0.008$ \\
\hline 6731 [S II] & $\ldots$ & $\ldots$ & $0.022 \pm 0.006$ & $0.022 \pm 0.006$ & $0.027 \pm 0.007$ & $0.022 \pm 0.006$ \\
\hline 7136 [Ar III] & $\ldots$ & $\ldots$ & $0.028 \pm 0.009$ & $0.028 \pm 0.009$ & $0.021 \pm 0.007$ & $0.017 \pm 0.005$ \\
\hline $\mathrm{C}(\mathrm{H} \beta) \operatorname{dex}$ & \multicolumn{2}{|c|}{$0.02 \pm 0.07$} & \multicolumn{2}{|c|}{$0.00 \pm 0.07$} & \multicolumn{2}{|c|}{$0.22 \pm 0.07$} \\
\hline$E W($ abs $) \AA$ & \multicolumn{2}{|c|}{$0.75 \pm 6.18$} & \multicolumn{2}{|c|}{$0.00 \pm 2.24$} & \multicolumn{2}{|c|}{$3.70 \pm 1.55$} \\
\hline$F(\mathrm{H} \beta)^{a}$ & \multicolumn{2}{|c|}{$12.1 \pm 0.7$} & \multicolumn{2}{|c|}{$13.7 \pm 0.7$} & \multicolumn{2}{|c|}{$13.6 \pm 0.7$} \\
\hline$E W(\mathrm{H} \beta) \AA$ & \multicolumn{2}{|c|}{$286 \pm 5$} & \multicolumn{2}{|c|}{$299 \pm 2$} & \multicolumn{2}{|c|}{$198 \pm 2$} \\
\hline \multirow[t]{2}{*}{ Rad. vel. $\mathrm{km} \mathrm{s}^{-1}$} & \multicolumn{2}{|c|}{$528 \pm 33$} & \multicolumn{2}{|c|}{$411 \pm 33$} & \multicolumn{2}{|c|}{$447 \pm 45$} \\
\hline & \multicolumn{2}{|c|}{ Knot $3, \mathrm{PA}=-57^{\circ}, \theta=0^{\prime} .8$} & \multicolumn{2}{|c|}{$\operatorname{Knot} 3, \mathrm{PA}=-57^{\circ}, \theta=1^{\prime \prime} .4$} & \multicolumn{2}{|c|}{ Knot $7, \mathrm{PA}=40^{\circ}, \theta=1^{\prime \prime} .2$} \\
\hline$\lambda_{0}(\AA)$ Ion & $F(\lambda) / F(\mathrm{H} \beta)$ & $I(\lambda) / I(\mathrm{H} \beta)$ & $F(\lambda) / F(\mathrm{H} \beta)$ & $I(\lambda) / I(\mathrm{H} \beta)$ & $F(\lambda) / F(\mathrm{H} \beta)$ & $I(\lambda) / I(\mathrm{H} \beta)$ \\
\hline 3727 [O II] & $0.656 \pm 0.034$ & $0.678 \pm 0.040$ & $0.656 \pm 0.033$ & $0.823 \pm 0.045$ & $1.085 \pm 0.082$ & $1.194 \pm 0.095$ \\
\hline $4101 \mathrm{H} \delta$ & $0.205 \pm 0.016$ & $0.278 \pm 0.028$ & $0.211 \pm 0.013$ & $0.249 \pm 0.021$ & $0.388 \pm 0.089$ & $0.413 \pm 0.109$ \\
\hline $4340 \mathrm{H} \gamma$ & $0.421 \pm 0.030$ & $0.474 \pm 0.038$ & $0.454 \pm 0.027$ & $0.503 \pm 0.032$ & $0.465 \pm 0.059$ & $0.484 \pm 0.094$ \\
\hline 4363 [O III] & $0.061 \pm 0.018$ & $0.060 \pm 0.018$ & $0.039 \pm 0.010$ & $0.042 \pm 0.011$ & $0.029 \pm 0.038$ & $0.031 \pm 0.040$ \\
\hline $4861 \mathrm{H} \beta$ & $1.000 \pm 0.016$ & $1.000 \pm 0.018$ & $1.000 \pm 0.009$ & $1.000 \pm 0.010$ & $1.000 \pm 0.038$ & $1.000 \pm 0.042$ \\
\hline 4959 [O III] & $0.438 \pm 0.026$ & $0.418 \pm 0.026$ & $0.412 \pm 0.022$ & $0.403 \pm 0.022$ & $0.212 \pm 0.029$ & $0.210 \pm 0.029$ \\
\hline 5007 [O III] & $1.329 \pm 0.069$ & $1.264 \pm 0.069$ & $1.270 \pm 0.064$ & $1.235 \pm 0.063$ & $0.600 \pm 0.032$ & $0.593 \pm 0.032$ \\
\hline $5876 \mathrm{He} \mathrm{I}$ & $0.094 \pm 0.012$ & $0.085 \pm 0.011$ & $0.088 \pm 0.008$ & $0.075 \pm 0.007$ & $0.118 \pm 0.018$ & $0.110 \pm 0.017$ \\
\hline 6548 [N II] & $\ldots$ & & $0.010 \pm 0.007$ & $0.008 \pm 0.005$ & $0.006 \pm 0.023$ & $0.005 \pm 0.021$ \\
\hline $6563 \mathrm{H} \alpha$ & $3.019 \pm 0.152$ & $2.690 \pm 0.154$ & $3.535 \pm 0.179$ & $2.750 \pm 0.152$ & $2.977 \pm 0.062$ & $2.683 \pm 0.062$ \\
\hline 6584 [N II] & $\ldots$ & $\ldots$ & $0.035 \pm 0.020$ & $0.027 \pm 0.016$ & $0.029 \pm 0.035$ & $0.026 \pm 0.032$ \\
\hline $6678 \mathrm{He} \mathrm{I}$ & $0.026 \pm 0.009$ & $0.023 \pm 0.008$ & $0.024 \pm 0.006$ & $0.019 \pm 0.004$ & $\ldots$ & $\ldots$ \\
\hline 6717 [S II] & $0.056 \pm 0.018$ & $0.050 \pm 0.016$ & $0.066 \pm 0.012$ & $0.051 \pm 0.009$ & $0.056 \pm 0.024$ & $0.050 \pm 0.021$ \\
\hline 6731 [S II] & $0.019 \pm 0.015$ & $0.017 \pm 0.014$ & $0.035 \pm 0.011$ & $0.027 \pm 0.009$ & $\ldots$ & $\ldots$ \\
\hline $\mathrm{C}(\mathrm{H} \beta) \operatorname{dex}$ & 0.10 & 0.07 & 0.32 & 0.07 & 0.14 & 0.07 \\
\hline$E W($ abs $) \AA$ & 3.20 & 0.61 & 0.25 & 0.63 & 0.05 & 5.71 \\
\hline$F(\mathrm{H} \beta)^{a}$ & 8.5 & 0.5 & 9.1 & 0.5 & 3.4 & 0.2 \\
\hline$E W(\mathrm{H} \beta) \AA$ & & & & & & 3 \\
\hline Rad. vel. $\mathrm{km} \mathrm{s}^{-1}$ & 501 & 36 & 447 & $=39$ & 309 & 93 \\
\hline
\end{tabular}

${ }^{a}$ In units of $10^{-16} \mathrm{ergs} \mathrm{s}^{-1} \mathrm{~cm}^{-2}$.

Fig. 1. As seen on the net $\mathrm{H} \alpha$ image, it has the form of an almost closed "circle" with a diameter of $\sim 270$ pc. Its SE sector is significantly brighter than the rest of this "circle" and consists of 3 adjacent knots. The brightest of them is quite elongated with the major axis oriented perpendicular to the arc to which it is connected. As discussed above, its starburst age is $\sim 4.5 \mathrm{Myr}$, which implies that the formation of the "ring"-like structures at both edges of DDO 68 have been synchronized by some global process.

The main issue to address based on our $V, R$, and $\mathrm{H} \alpha$ images is the age of the oldest visible stellar population. We built surface brightness profiles (SBPs) for $V, R$ images applying aperture photometry in circular apertures centred at the point near the geometrical centre of the main DDO 68 body (with J2000 coordinates of 09:56:45.8 +28:49:27). They are shown in Fig. 3.

The nebular emission in DDO 68 is not as strong and widespread as in SBS 0335-052 and I Zw 18. However, it somewhat affects the observed colours on the significant part of the colour profile in Fig. 3. The contribution of various features superimposed on the smooth distribution of the assumed underlying light is seen clearly on both SBPs (top panel) and 
Table 4. Abundances in Knots 1, 2 and 3 of DDO 68.

\begin{tabular}{lcc|ccc|cc}
\hline \hline & \multicolumn{2}{c|}{ Knot 1} & \multicolumn{3}{c|}{ Knot 2} & \multicolumn{2}{c}{ Knot 3} \\
\cline { 2 - 7 } Value & $\mathrm{PA}=-90^{\circ}$ & $\mathrm{PA}=0^{\circ}$ & $\mathrm{PA}=-57^{\circ}$ & $\mathrm{PA}=0^{\circ}$ & $\mathrm{PA}=-57^{\circ}$ & $\mathrm{PA}=-57^{\circ}$ & $\mathrm{PA}=-57^{\circ}$ \\
& $\theta=00^{\prime} 8$ & $\theta=1^{\prime \prime} 6$ & $\theta=00^{\prime} 8$ & $\theta=1^{\prime \prime} 6$ & $\theta=1^{\prime \prime} .4$ & $\theta=0.8$ & $\theta=1^{\prime \prime} 4$ \\
\hline$T_{\mathrm{e}}(\mathrm{OIII})\left(10^{3} \mathrm{~K}\right)$ & $17.01 \pm 1.32$ & $18.89 \pm 1.33$ & $19.33 \pm 4.27$ & $19.24 \pm 1.90$ & $20.13 \pm 2.07$ & $25.30 \pm 5.92$ & $20.28 \pm 3.28$ \\
$T_{\mathrm{e}}(\mathrm{OII})\left(10^{3} \mathrm{~K}\right)$ & $14.64 \pm 1.08$ & $15.34 \pm 1.02$ & $15.48 \pm 3.18$ & $15.45 \pm 1.42$ & $15.72 \pm 1.48$ & $16.73 \pm 2.61$ & $15.77 \pm 2.33$ \\
$T_{\mathrm{e}}(\mathrm{SIII})\left(10^{3} \mathrm{~K}\right)$ & $15.82 \pm 1.1$ & $17.38 \pm 1.11$ & $17.73 \pm 3.54$ & $17.66 \pm 1.58$ & $18.41 \pm 1.72$ & $22.70 \pm 4.92$ & $18.53 \pm 2.72$ \\
& & & & & & \\
$\mathrm{O}^{+} / \mathrm{H}^{+}\left(\times 10^{-5}\right)$ & $0.177 \pm 0.036$ & $0.442 \pm 0.078$ & $0.369 \pm 0.193$ & $0.358 \pm 0.086$ & $0.602 \pm 0.145$ & $0.431 \pm 0.159$ & $0.616 \pm 0.229$ \\
$\mathrm{O}^{++} / \mathrm{H}^{+}\left(\times 10^{-5}\right)$ & $1.489 \pm 0.287$ & $1.205 \pm 0.203$ & $0.944 \pm 0.479$ & $0.965 \pm 0.222$ & $0.886 \pm 0.208$ & $0.470 \pm 0.230$ & $0.686 \pm 0.251$ \\
$\mathrm{O}^{+++} / \mathrm{H}^{+}\left(\times 10^{-5}\right)$ & $0.052 \pm 0.015$ & $0.068 \pm 0.016$ & $0.192 \pm 0.074$ & $0.309 \pm 0.110$ & $0.159 \pm 0.053$ & $\ldots$ & $\ldots$ \\
$\mathrm{O} / \mathrm{H}\left(\times 10^{-5}\right)$ & $1.716 \pm 0.289$ & $1.715 \pm 0.218$ & $1.505 \pm 0.521$ & $1.632 \pm 0.262$ & $1.648 \pm 0.260$ & $0.901 \pm 0.280$ & $1.302 \pm 0.339$ \\
$12+\log (\mathrm{O} / \mathrm{H})$ & $7.23 \pm 0.07$ & $7.23 \pm 0.06$ & $7.18 \pm 0.16$ & $7.21 \pm 0.07$ & $7.22 \pm 0.07$ & $6.95 \pm 0.14$ & $7.11 \pm 0.11$ \\
\hline
\end{tabular}

Table 5. Starburst ages in DDO 68.

\begin{tabular}{ccc}
\hline \hline Region & $E W(\mathrm{H} \beta)$ & Age $(\mathrm{Myr})$ \\
\hline Knot 1 & $117 \pm 5$ & 4.0 \\
Knot 2 & $250 \pm 30$ & 3.0 \\
Knot 3 & $76 \pm 3$ & 5.0 \\
Knot 4 & $206 \pm 32$ & 3.5 \\
Knot 6 & $47 \pm 3$ & 7.0 \\
Knot 7 & $87 \pm 4$ & 4.5 \\
\hline
\end{tabular}

Table 6. Balmer absorptions in Knot 5.

\begin{tabular}{cr}
\hline \hline$\lambda_{0}$, name & $E W(\AA)$ \\
\hline $4102 \mathrm{H} \delta$ & $5.8 \pm 0.5$ \\
$4340 \mathrm{H} \gamma$ & $4.4 \pm 0.4$ \\
$4861 \mathrm{H} \beta$ & $4.4 \pm 0.4$ \\
$6563 \mathrm{H} \alpha$ & $-4.6 \pm 0.1$ \\
\hline
\end{tabular}

Minus in $E W(\mathrm{H} \alpha)$ means emission.

the colour radial profile (bottom panel). The strongest features are seen clearly in the $\mathrm{H} \alpha$ image (Fig. 1). The nebular emission shows the most contrast in the two ring-like regions at the $\mathrm{N}$ and $\mathrm{S}$ edges of the galaxy. They appear on both the SBP and the colour radial distribution as clear peaks at $R \sim 40,55,80$, and $100^{\prime \prime}$. Their contribution within the main body $\left(R \leq R_{\mathrm{opt}}=\right.$ $\left.31^{\prime \prime} 5\right)$ and adjacent regions is less pronounced, but is still significant till the radial distance of $R \sim 34$ ".

We fitted the $V, R$ SBPs by the "minimal" exponential disks, using for this only the ranges in radial coordinates where the contribution of the superimposed emission is minimal/absent, namely $20^{\prime \prime}<R<40^{\prime \prime}$ and $60^{\prime \prime}<R<70^{\prime \prime}$. The derived scalelengths of the disks in $V$ and $R$-bands do not differ significantly within their uncertainties. Their mean value is $\langle\alpha\rangle=15^{\prime \prime} 3 \pm 0.3$, corresponding to the linear value of $482 \pm 10 \mathrm{pc}$. We therefore accept the hypothesis that the underlying light is described by the unique exponential disk. The central surface brightnesses of the mean fitted disk are $\mu_{V}^{0}=$ $22.56 \mathrm{mag} \operatorname{arcsec}^{-2}$ and $\mu_{R}^{0}=22.34 \mathrm{mag} \operatorname{arcsec}^{-2}$ with a total error of $\sim 00^{\mathrm{m}} 06$. The $(V-R)^{0}$ colour radial profile along with the derived disk colour (solid line) are shown in the bottom panel of Fig. 3.

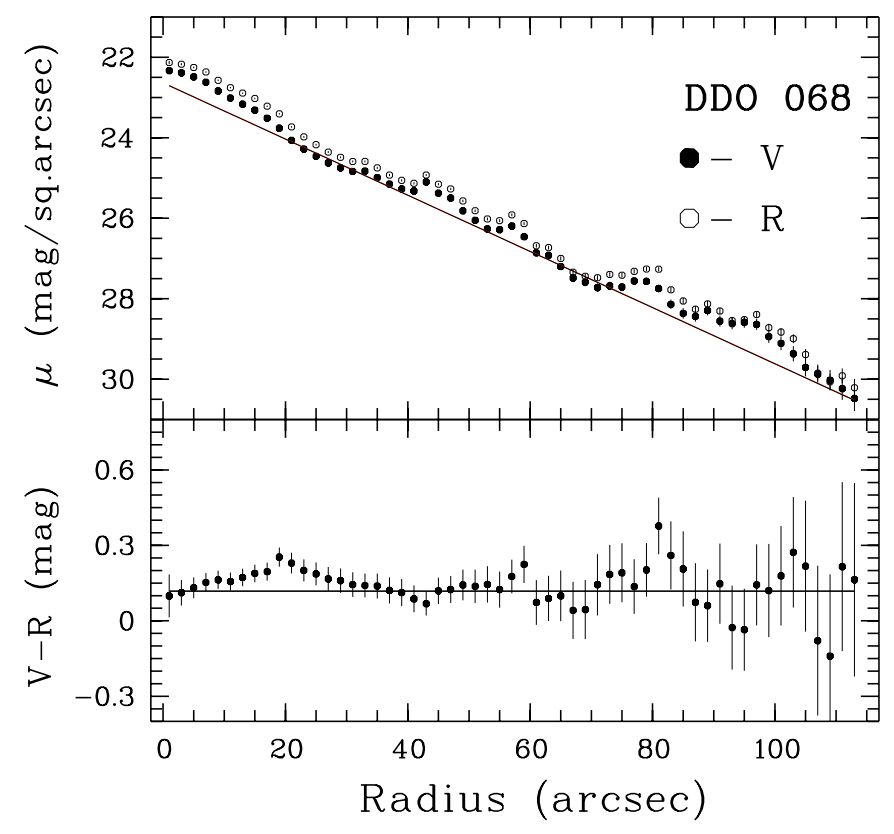

Fig. 3. Top: the $V$ and $R$ surface brightness radial profiles (SBP) of DDO 68 obtained in circular apertures with a step of $2^{\prime \prime}$. Shown error bars correspond to $2 \sigma$ uncertainties. The errors of the photometric system are not included. The fit by an underlying exponential disk is shown only for $V$-band SBP. Additional light seen at $R \sim 40^{\prime \prime}-60^{\prime \prime}$ corresponds to Knots No. 1-5. Additional light for $R>70^{\prime \prime}$ corresponds to Knot No. 7 and the southern tail. Bottom: respective radial profile of $(V-R)^{0}$ colour with the correction of $-0{ }^{\mathrm{m}} 09$ for the zeropoint shift (see Sect. 3.4) and for the Galaxy extinction. Error bars correspond to $2 \sigma$ uncertainties without the errors of the photometric system. The $(V-R)^{0}$ colour of the exponential disk of 0.12 is shown by the solid line. Its rms uncertainty is $\sigma=0$. 04 .

Total magnitudes of DDO 68 derived by the integration within a polygon mask including all galaxy light, with the removal of foreground stars and background galaxies, are as follows: $V=14.46 \pm 0.05, R=14.20 \pm 0.05$, with the resulting $(V-R)_{\text {total }}=0.26 \pm 0.07$. The relatively large errors appear from the uncertainties of the coefficients of the photometrical system, built on observations of the calibration field near QSO FBQ $0951+263$. The total flux of DDO 68 in the $\mathrm{H} \alpha$-line, 
derived within the same polygon mask, is $(1.73 \pm 0.09) \times$ $10^{-13} \mathrm{erg} \mathrm{cm}^{-2} \mathrm{~s}^{-1}$, which in turn corresponds to a total $\mathrm{H} \alpha$ luminosity of $0.88 \times 10^{39} \mathrm{erg} \mathrm{s}^{-1}$. We did not transfer this value to the traditional star formation rate (SFR), since for young starbursts the SFR derived through the commonly used formulas is highly uncertain (see Weilbacher \& Fritze-v. Alvensleben (2001). However, we compare this and other global parameters with those in I Zw 18 and SBS 0335-052 in the discussion.

Since the "minimal" disk describes the widespread underlying stellar population over the whole galaxy volume, its colours can be considered as indicating the oldest stellar population in the galaxy. This colour $(V-R)_{\text {disk }}=0 \mathrm{~m} \cdot 22$, with the rms error of 0.07 due to the photometric system uncertainties. A small correction of $\sim 0^{\mathrm{m}} 012$ for the Galaxy extinction should also be applied before comparison with the evolutionary tracks of PEGASE. 2 models (Fioc \& Rocca-Volmerange 1997, 1999). We took the models for the metallicity of $z=0.0004$, the nearest to that of DDO 68. The Salpeter IMF is accepted with $M_{\text {low }}$ and $M_{\text {up }}$ of $0.1 M_{\odot}$ and $120 M_{\odot}$, respectively. Various modes of SF (instantaneous and continuous) result in different age constraints on the oldest visible stellar population.

Taken at face value, the disk colour $\left((V-R)^{0} \sim 0.21\right)$ with the cited error of $\sigma_{(V-R)}=0.07$ corresponds to either 0.65-1.8-4.5 Gyr-old (lower and upper limits correspond to $-1 \sigma$ and $+1 \sigma$ ) stellar clusters for the continuous SF with constant SFR or to 0.11-0.12-1.0 Gyr-old cluster for the instantaneous starburst.

\subsection{Properties of the young star cluster in Knot 5}

The young star cluster (YSC) related to Knot 5 is one of a few known with such low metallicity that can be directly studied in great detail. The others include, e.g., some stellar clusters containing WR stars in I Zw 18 (Brown et al. 1996) or unresolved with HST and VLT young clusters in SBS 0335-052 E (Thuan et al. 1997; Plante \& Savage 2002). Apart from the difference in metallicity by a factor of $\sim 30$, it resembles the young super star cluster in the bubble complex of NGC 6946 very well (Elmegreen et al. 2000; Efremov et al. 2002; Larsen et al. 2001), which is situated at a similar distance of $\sim 6 \mathrm{Mpc}$. The latter was successfully observed with the HST and Keck HIRES to resolve its stellar content and velocity dispersion and to address its evolutionary status. While for the YSC in DDO 68, such observations are feasible in the future as well, here we summarise the properties that emerge from current observational data.

We can estimate the characteristic size of the Knot 5 cluster by assuming its intrinsic intensity distribution to be Gaussian with the respective $F W H M_{\mathrm{YSC}}$. Subtracting (quadratically) the PSF $F W H M\left(00^{\prime} 8\right)$ from the observed $F W H M\left(1{ }^{\prime \prime} 12\right)$, we obtained the $F W H M_{\text {YSC }}$ intrinsic diameter of $\sim 0{ }^{\prime} 8$. The respective linear diameter of this cluster is $25 \mathrm{pc}$. For comparison, compact young clusters in SBS 0335-052 E, unresolved on the HST images (the PSF $F W H M=0 ! 2$ ) have diameters less than $50 \mathrm{pc}$ (Thuan et al. 1997). The further analogy of the DDO 68 young star cluster with the mentioned above in NGC 6946 extends to their linear size. As described by Larsen et al. (2001), this cluster has a core with a radius of $1.3 \mathrm{pc}$, surrounded by an extended halo with the power-law luminosity profile. The halo half-light radius is $13 \mathrm{pc}$, close to half of the $F W H M$ of the light profile of Knot 5 in DDO 68. With the age of 12-15 Myr (Efremov et al. 2002), the young star cluster in NGC 6946 is, however, significantly younger.

The total $V=19^{\mathrm{m}} .40$ of the YSC in DDO 68 corresponds to the absolute magnitude $M_{V}^{0}=-9.66$. By comparing this $M_{V}^{0}$ with that from the PEGASE. 2 model for instantaneous starbursts of the metallicity $z=0.0004$ cluster with the Salpeter IMF for the age $T=22.5 \mathrm{Myr}\left(M_{V}=2.30\right.$ per $\left.1 M_{\odot}\right)$, we derived the mass of the young cluster of $M_{\mathrm{YSC}}=6.0 \times 10^{4} M_{\odot}$. This cluster is the most massive in the "Northern ring". The brightest among the others, Knot 1, with $V \sim 20^{\mathrm{m}} 3$, has a star cluster mass of $4.4 \times 10^{3} M_{\odot}$.

The measured colour of Knot $5(V-R)^{0}=0^{\mathrm{m}} \cdot 11 \pm 0.01 \pm$ 0 m 07 , where the second component of the error comes from the photometric system, is redder by 0.09 than predicted by the PEGASE. 2 model for its age $\left((V-R)=0{ }^{\mathrm{m}} 02\right)$. This is most probably related to some systematics in the zero-points of the constructed photometric system (see Sect. 3.4).

\subsection{Colour correction and improved age estimates}

The main uncertainties of the derived $(V-R)$ colours of the underlying disk and of the estimate of the age of the oldest visible stellar population are related to the zero-points uncertainty of the constructed photometrical system $\left(\sigma_{(\mathrm{V}-\mathrm{R})}=0.07\right)$. Having some independent observational data for DDO 68, we can now diminish this effect. For this we used two different approaches.

First, we used the spectral information (EWs of Balmer absorption and emission lines) for Knot 5 - the young compact star cluster in the northern "ring", which implies its age of $\sim 22.5 \mathrm{Myr}$. Then, as shown at the end of the previous section, the observed $(V-R)$ colour is 0.09 redder than predicted by the PEGASE. 2 models for its respective age. This implies the presence of the additive small shift of 0.09 in the $(V-R)$ zeropoint due to the photometric system uncertainties.

Second, there is an independent indication of the reality of the $(V-R)$ colour shift found above. This comes from the comparison of our measured $(V-R)_{\text {total }}=0.26$ and the colour $(B-V)_{\text {total }}=0.17$ from Karachentsev et al. (2004) in Table 7, with $(B-R)_{\text {total }}=0.32 \pm 0.03$ from Hopp \& SchulteLadbeck (1995). Combining the former, our value with the cited $(B-V)_{\text {total }}$ led to $(B-R)_{\text {total }}=0.43$, while using the above correction brought this parameter to value of 0.34 , significantly more consistent with that of Hopp \& Schulte-Ladbeck.

Now we apply this correction of the $V-R$ zero-point and the respective reduction of its uncertainty roughly to 0.04 from comparison with the Hopp and Schulte-Ladbeck $(B-R)_{\text {total }}$ to the colours of the underlying population. The corrected $(V-R)^{0}$ colour of the underlying disk is $0 \mathrm{~m} \cdot 12 \pm 0 \mathrm{~m} 04$. This in turn corresponds (in the frame of the same PEGASE.2 models as above) to the ages of 200-450-900 Myr for the case of continuous SF with constant SFR, or to the ages of 100-105-115 Myr for the case of instantaneous starburst. The lower and upper limits correspond, as above, to $-1 \sigma$ and $+1 \sigma$ in the $(V-R)$ colour. 
Table 7. Main parameters of DDO 68 and I Zw 18

\begin{tabular}{lrr}
\hline \hline Parameter & DDO 68 & I Zw 18 \\
\hline Type & Im/BCD & BCD \\
$B_{\text {tot }}$ & 14.60 & $16.12^{2}$ \\
$(B-V)_{\text {tot }}$ & 0.17 & $0.11^{2}$ \\
$(V-R)_{\text {tot }}$ & $0.17^{3}$ & $0.12^{2}$ \\
$V_{\text {Hel }}\left(\mathrm{km} \mathrm{s}^{-1}\right)$ & 502 & $751^{4}$ \\
$D(\mathrm{Mpc})$ & $6.5^{3}$ & $15^{8}$ \\
$E(B-V)^{5}$ & 0.018 & 0.032 \\
$M_{B}^{0}$ & -14.33 & -14.89 \\
Angular size $\left(^{\prime \prime}\right)$ & $103 \times 38^{3}$ & $23 \times 13^{2}$ \\
Optical size $(\mathrm{kpc})$ & $3.2 \times 1.2^{3}$ & $1.5 \times 0.8$ \\
$12+\log (\mathrm{O} / \mathrm{H})$ & 7.21 & $7.17^{6,7}$ \\
$\mathrm{H} \alpha$ flux* & $1.73^{3}$ & $3.52^{9}$ \\
$\mathrm{H} \mathrm{I} \mathrm{flux}$ & 26.7 & $2.97^{4}$ \\
$W_{50}\left(\mathrm{~km} \mathrm{~s}^{-1}\right)$ & 78 & $49^{4}$ \\
$W_{20}\left(\mathrm{~km} \mathrm{~s}^{-1}\right)$ & 85 & $84^{4}$ \\
$M(\mathrm{HI})\left(10^{8} M_{\odot}\right)$ & 2.6 & 1.6 \\
$M(\mathrm{HI}) / L_{B}^{* * *}$ & 1.85 & 1.29 \\
\hline
\end{tabular}

$B_{\text {tot }}$ - total blue magnitude; $M_{B}^{0}$ - absolute blue mag. $L_{B}$ - total blue luminosity. ${ }^{*}$ Units of $10^{-13} \mathrm{erg} \mathrm{s}^{-1} ;{ }^{* *}$ Units of $\mathrm{Jy} \mathrm{km} \mathrm{s}^{-1} ;{ }^{* * *}$ in units of $\left(M / L_{B}\right)_{\odot}{ }^{\ddagger} a \times b$ at the surface brightness $\mu_{B}=25 \mathrm{mag} \mathrm{arcsec}^{-2}$. References: ${ }^{1}$ DDO 68 parameters without reference are from Karachentsev et al. (2004); ${ }^{2}$ Papaderos et al. (2002); ${ }^{3}$ this paper; ${ }^{4}$ van Zee et al. (1998; ${ }^{5}$ Schlegel et al. (1998); ${ }^{6}$ Izotov et al. (1999); ${ }^{7}$ Kniazev et al. (2003); ${ }^{8}$ Izotov \& Thuan (2004); ${ }^{9}$ Gil de Paz et al. (2003).

Applying the above correction of $(V-R)$, we somewhat improve the accuracy of the derived ages of the old population. However, due to the well known degeneracy of the colour evolutionary tracks for different SF laws, the estimated ages are still uncertain by a factor of $\sim(2-8)$, if based only on the optical colours of unresolved stars, depending on the accepted SF law. Besides, the use of the other optical-NIR colours is important to check the results suggested by the study of only $V-R$ colour.

The most reliable age estimates can be obtained from the colour-magnitude diagrams (CMD) of resolved stars. If the galaxy is indeed young, no RGB stars will be seen in the CMD, as in the case of I Zw 18 (Izotov \& Thuan 2004). For the distance modulus of DDO 68 equal to 29.0 (for $D=6.5 \mathrm{Mpc}$ ), stars on the tip of RGB should have $V=25.0$ and $I=23$. 8 .

\section{Discussion and conclusions}

\subsection{Properties of DDO 68 in comparison to I Zw 18}

The weighted mean $12+\log (\mathrm{O} / \mathrm{H})=7.21 \pm 0.03$ for DDO 68 is very close to that in $\mathrm{I} \mathrm{Zw} 18$. Besides, DDO 68 is close to I Zw 18 on several other parameters. In particular, its absolute blue magnitude $M_{B}$ is only 0 . 56 fainter. Thus, both objects are very close on the $L-Z$ diagram and, together with SBS 0335-052 E and W, comprise a group of galaxies significantly deviating from the general $L-Z$ relation for BCGs (see, e.g., Pustilnik et al. 2003a). Such deviations are also noticed for several luminous BCGs with $12+\log (\mathrm{O} / \mathrm{H}) \sim 8.0$ (e.g., Kunth \& Östlin 2000), which are old galaxies, so the strong deviation from the general $L-Z$ relation is not an exclusive property of the most metal-deficient BCGs. However, the objects of this small group deviate as well on the $L-Z$ diagram from the region where the majority of known XMD galaxies are situated (see Kniazev et al. 2003). Based on this large deviation, Kniazev et al. (2003) suggest that the small XMD subgroup of I Zw 18 and its 'cousins' are truly young galaxies. A similar conclusion was formulated for the same small subgroup of XMD galaxies by Guseva et al. (2003), based on the very blue $(V-I)$ colours of their LSB components.

The $M(\mathrm{HI}) / L_{\mathrm{B}}$ ratio for DDO 68 is also sufficiently high (1.85), and is a factor of $\sim 1.4$ higher than for I $\mathrm{Zw} 18$. Its total H I mass $\left(2.6 \times 10^{8} M_{\odot}\right)$ is a factor of 1.6 higher than the H I mass of I Zw 18. Its integrated colour $(B-V)_{\text {tot }}^{0}=0.17$ (Makarova \& Karachentsev 1998) is close to that of I Zw 18 (0. 12 ). Some other properties of these two galaxies are collected in Table 7 for comparison. Direct comparison of the current SFR (based on the $\mathrm{H} \alpha$-luminosity) of DDO 68 and other similar galaxies is not meaningful, since this SFR changes significantly on a short timescale. However the specific values of $\mathrm{H} \alpha$-luminosity related either to the blue luminosity or to the total H I mass are of interest. For further comparison, we take $\mathrm{F}(\mathrm{H} \alpha)$ of I Zw 18 from Gil de Paz et al. (2003).

In DDO 68 these parameters are significantly lower than for I $\mathrm{Zw} \mathrm{18,} \mathrm{indicating} \mathrm{a} \mathrm{lower} \mathrm{current} \mathrm{production} \mathrm{of} \mathrm{the} \mathrm{ion-}$ izing radiation. Namely, $L(\mathrm{H} \alpha) / L_{\mathrm{B}}$ is $\sim 0.005$ for DDO 68 and 0.035 - for I Zw 18. Similar, $L(\mathrm{H} \alpha) / M(\mathrm{HI})$ is 0.0016 (in units $\left.\left(L_{B} / M\right)_{\odot}\right)$ for DDO 68 and 0.028 - for I Zw 18. Interestingly, on these parameters DDO 68 is closer to SBS 0335-052 W, where these ratios are $L(\mathrm{H} \alpha) / L_{\mathrm{B}}=0.02$ and $L(\mathrm{H} \alpha) / M(\mathrm{HI})=$ 0.0027 ( $\mathrm{H} \alpha$-flux is from Pustilnik et al. 2004a). It was noticed that based on its properties, SBS 0335-052 W is not a typical blue compact galaxy, but instead resembles dIrr, which based on the strength of the current "starburst" is also correct for DDO 68. The close similarity of principal observational properties of DDO 68 and I Zw 18 suggests that DDO 68 may also be a young galaxy, that is it contains no stellar population with ages more than $1 \mathrm{Gyr}$.

\subsection{Optical and $\mathrm{H}$ I morphology and their implications}

The strongly disturbed optical morphology of DDO 68 is difficult to understand since the galaxy is rather distant from any potential disturber. The luminous galaxies are at least at $\sim 2.0 \mathrm{Mpc}$ (NGC 2683 with $M_{B}=-20.4$ at $D=7.7 \mathrm{Mpc}$ ) and $\sim 2.5 \mathrm{Mpc}$ (NGC 2903 with $M_{B}=-21.0$ at $D=8.9 \mathrm{Mpc}$, Karachentsev et al. 2004). The nearest known galaxy, a dwarf UGC 5427, with $M_{B}=-14.5$ at $\sim 200 \mathrm{kpc}$ in projection and with very close radial velocity, is too low-mass object to affect DDO 68. The neutral gas morphology and kinematics, as revealed by H I maps with 13 ". 5 angular resolution by Stil \& Israel $(2002 \mathrm{a}, \mathrm{b})$, are also well disturbed with several maxima of density and with several holes with linear sizes of the order of $1 \mathrm{kpc}$.

One could consider two options to understand the optical morphology of DDO 68. The first is related to the strong disturbance by some separate, massive, poorly visible (or 
optically invisible) object. This could be a very low surface brightness galaxy, or an intergalactic H I cloud, similar to the case of HI 1225+01 (Salzer et al. 1991; Chengalur et al. 1995). The second option is related to an advanced merger, when two merging objects are already not seen as separate entities. However, the morphology of the joint material clearly indicates outflows/plumes as the characteristic features created by strong interaction. The importance of the merger channel for starbursts in BCGs was emphasised, e.g., by Östlin et al. (2001). Of course, the combined option is also possible: for example, a well-advanced merger with an intergalactic $\mathrm{H}$ I cloud.

To distinguish the above options, the available H I maps are very useful. We also used overlays of the H I density map on the DDO 68 I-band and $\mathrm{H} \alpha$ images, along with detailed data on $\mathrm{H} \mathrm{I}$ velocity dispersion in the Ph.D. thesis of Stil (1999). Analysis of all these data leads to the next conclusions. First, there is no indication of the presence of any separate massive $\mathrm{HI}$ object in the vicinity of DDO 68. Second, there is the large asymmetry of HI density distribution relative to the optical body of the galaxy. The H I density map can be divided conditionally into two curved chains of dense matter stretching roughly from North to South, with the typical separation between the chains of $0.5-1.0(1-2 \mathrm{kpc})$. The optical body, including the Northern "ring" and the Southern "tail", coincides roughly with the Eastern H I chain, while practically no optical counterparts are seen for the Western H I chain (at least, on the SB level, comparable with the optical light seen along the Eastern H I chain).

The large asymmetry between the distributions of H I mass and the optical light suggests that the Western part of the H I body is unrelated directly to the optical galaxy and represents some external H I cloud in the process of merging with a gasrich massive object, in which some star formation has already occurred and is still taking place. The elevated velocity dispersion in the four densest regions (by a factor of 2 to 3 relative to the undisturbed regions, which have $\sigma_{v}=6-9 \mathrm{~km} \mathrm{~s}^{-1}$ ), all them being displaced to the Eastern half of the whole $\mathrm{H}$ I cloud, gives additional evidence of strong gas agitation due to the ongoing merging, as suggested by Elmegreen et al. (1993). If this hypothesis is correct, DDO 68 can represent the well-advanced stage of merging observed in the system HI $1225+01$. The metallicity of gas in the two merging components can differ substantially, so the complex process of the gas mixing can create significant spatial inhomogeneities in the ISM metal content of DDO 68.

While the WSRT DDO 68 H I map does not show the extended tidal tails characteristic of many mergers, this is not an argument against the merger case. However, the tails are not likely to be seen because the available maps are relatively shallow, with the lowest $\mathrm{HI}$ density contour of $5 \times 10^{20}$ atom $\mathrm{cm}^{-2}$. The extended tidal tails in the well-advanced mergers like those in NGC 7252, NGC 3921, NGC 3526 (e.g., Hibbard et al. 2001), are visible at the H I column densities of (one - a few) $10^{19}$ atom $\mathrm{cm}^{-2}$. Thus, in the frame of the merger hypothesis, we predict that the deeper H I mapping of DDO 68 will uncover the extended gas tidal tails.

We also mention a more exotic variant of the first option, when the strong interaction of a gas-rich dwarf took place with a completely dark galaxy, which is a massive DM halo with the baryon mass fraction well below that typical of galaxies (Trentham et al. 2001). As these authors suggest, this can be the trigger of starbursts in many isolated BCGs. However, to check this hypothesis observationally, they need to have very clear-cut predictions, since at first approximation it is difficult to distinguish this case from well-advanced merger.

\subsection{Chemical evolution and environment}

In the frame of Cold Dark Matter cosmology the galaxies formed in the regions of the very low density (voids) should be of lower mass and retarded in their formation epoch in comparison to the more massive galaxies from the average and the higher density regions (e.g., Gottlöber et al. 2003). Besides, in the frame of the widely accepted hierarchical galaxy formation scenario, their life cycles can differ quite a lot from the more common galaxies. Namely, for a selected galaxy, the probability of interactions and mergers during the cosmological time is scaled down in voids with the galaxy density as $\rho_{\text {galaxy }}^{+1}$. Therefore, one could expect that some (small) fraction of void galaxies can survive in their nascent state, avoiding any significant interaction.

It is probably not by chance that one of the most metal-poor galaxies, DDO 68, is found in the very low-density "normal" galaxy environment. Another XMD galaxy HS 0822+3542 is situated near the centre of the same Lynx-Cancer void (Pustilnik et al. 2003b). At least one more dwarf galaxy with very low metallicity, KISSB $23=$ KUG 0937+298 $(12+$ $\log (\mathrm{O} / \mathrm{H})=7.65$, Lee et al. 2004) is also situated within the same void, only at $\sim 650 \mathrm{kpc}$ from DDO 68 .

The nearest neighbour of DDO 68 is UGC 5427 ( $\mathrm{Sdm}$, $B \sim 14^{\mathrm{m}} .9$ ) at the angular distance of $\sim 1.8^{\circ}$, corresponding to $\sim 200 \mathrm{kpc}$ in projection. Its radial velocity is only $7 \mathrm{~km} \mathrm{~s}^{-1}$ lower than that of DDO 68. Karachentsev et al. (2004) accept distances for DDO 68 and UGC 5427 derived by the method of the brightest stars (accuracies of 25\%), of 5.9 and $7.1 \mathrm{Mpc}$, respectively. We suggest that both galaxies belong to the same "filament" and the distance in between is on the order of their projected distance. Therefore, we accept their common radial distance as a mean of their individual determinations, consistent each to other: $D_{\text {mean }}=6.5 \mathrm{Mpc}$.

As discussed by Pustilnik et al. (2004a), a substantial fraction of XMD galaxies are found either at the outskirts of galaxy aggregates (e.g., SBS 0335-052 E,W and Dw 1225+0152), or in voids (e.g., HS $0822+3542$, HS 2236+1344, HS 0837+4717). While the statistics and properties of void galaxy population are still not well known, it is assumed from general consideration that the significantly reduced frequency of galaxy interactions in voids provides the most favourable conditions to allow for the stablest gas protogalaxies to survive as purely gas objects. If such very stable protogalaxies do exist in voids, they can be detected either as purely gas bodies through the blind H I surveys or through the Ly- $\alpha$ absorption of a background UV-bright object (e.g., Manning 2002), or even as young galaxies, if they recently have 
experienced the strong gravitational disturbance due to close collision with sufficiently massive objects.

Therefore, on one hand, it is not surprising that many "void" XMD galaxies studied by us (a paper in preparation) show clear evidence of interactions and merging. This can be a kind of selection effect for the superstable objects, when starbursts in unevolved galaxies occur only due to the strong external disturbance. The case of DDO 68 is additional evidence for the common mode of such starbursts in the XMD galaxies situated in the very low density environment.

On the other hand, as explained by Kunth \& Sargent (1986), even the first starburst in such a retarded object, due to the effective self-pollution in the young $\mathrm{H}$ II regions, will be observed with a heavy element content comparable to $Z \sim Z_{\odot} / 30$ for the starburst ages larger than $3 \mathrm{Myr}$.

\subsection{Conclusions}

Summarising the observational data and the discussion above, we have drawn the following conclusions:

- The oxygen abundances in three H II regions at the northern edge of the dwarf galaxy DDO 68 are close one to another with the weighted mean of $12+\log (\mathrm{O} / \mathrm{H})=7.21 \pm$ 0.03 dex.

- DDO 68 is one of the three galaxies (I $\mathrm{Zw} 18$ and SBS 0335-052 W) with the lowest ISM metallicity, and the nearest galaxy with the same low metallicity (twice closer than I Zw 18).

- The deep photometry of DDO 68 indicates that its the reddest colours outside the central bright region and the regions of current/recent SF (where no nebular emission is seen) are very blue $\left((V-R)^{0}=0.12 \pm 0\right.$. 04$)$. Their comparison with the PEGASE. 2 models for the evolving stellar clusters (with metallicity of $z=0.0004$ ) implies that the oldest visible stars contributing to the light of the LSB underlying disk have ages either of 100-115 Myr. in the case of instantaneous starburst, or of 200-900 Myr, in the case of continuous star formation with the constant SFR. Thus, $V-R$ data indicate the possible youth of DDO 68. The data on other colours, as well on CMDs, are necessary, however, to check and strengthen this conclusion.

- The young star clusters (related, in particular to Knots 1 and 5 in the "Northern ring") with the masses of (0.4-6.0) $\times$ $10^{4} M_{\odot}$ are the nearest among all known such objects with the lowest metallicity. They are probably the best known clusters for which the direct spectroscopic study of individual massive stars with $Z \sim Z_{\odot} / 30$ will be possible with the future giant ground-based and space telescopes. Study of their structure and dynamics is currently feasible with the HST and the high resolution spectroscopy at large telescopes.

- The optical morphology of DDO 68 indicates recent interaction despite no disturbing galaxy is evident in its surrounding. The most probable interpretation of this galaxy's properties is a well-advanced merging of two gas-rich objects.
- DDO 68 is situated in the region with the low density of luminous galaxies near the rim of a nearby small Lynx-Cancer void. Apart from this galaxy, two more XMD dwarfs have been discovered in this void: HS $0822+3542$ and KISSB 23. Several other XMD galaxies are found in other voids. This is consistent with theoretical expectations that dwarfs in the regions with the very low density of luminous galaxies (and the very reduced density of low-mass galaxies) may form/evolve with significant delay. This opens a new direction in searching for local young galaxies.

Acknowledgements. The authors are pleased to thank V. Afanasiev and A. Moiseev for the help in the organization and preparation of observations with SCORPIO, Y. Izotov for useful comments, and J. Stil for kindly providing his Ph.D. thesis. We appreciate the criticism and suggestions of the referee, D. Kunth, which helped to improve the paper. We acknowledge the partial support from Russian state program "Astronomy". This research made use of the NASA/IPAC Extragalactic Database (NED), which is operated by the Jet Propulsion Laboratory, California Institute of Technology, under contract with the National Aeronautics and Space Administration. Use of the Digitized Sky Survey (DSS-II) is gratefully acknowledged.

\section{References}

Afanasiev, V. L., \& Moiseev, A. V. 2005, Astron. Lett. 31, 193

Aller, H. L., 1984, Physics of Thermal Gaseous Nebulae (Dordrecht: Reidel)

Alloin, D., Bergeron, J., \& Pelat, D. 1978, A\&A, 70, 141

Asplund, M., Grevesse, N., Sauval, A. J., Allende Prieto, C., \& Kiselman, D., 2004, A\&A, 417, 751

Bohlin, R. C. 1996, AJ, 111, 1743

Brown, T. M., Heap, S. R., Hubeny, I., Lanz, T., \& Lindler, D. 2002, ApJ, 579, L75

Chengalur, J. N., Giovanelli, R., \& Haynes, M. P., 1995, AJ 109, 2415

Duc, P.-A., \& Mirabel, I. F. 1999, in IAUS 186: Galaxy Interactions at Low and High Redshift, Kyoto 1997, ed. J. E. Barnes and D. B. Sanders., 61

Efremov, Y., Pustilnik, S., Kniazev, A., et al. 2002, A\&A, 389, 855

Elmegreen, B. G., Kaufman, M., Thomasson, M. 1993, ApJ, 412, 90

Elmegreen, B. G., Efremov, Y. N., \& Larsen, S. 2000, ApJ, 535, 748

Elmegreen, B. G., Palous, J., \& Ehlerova, S. 2004, MNRAS, 334, 693

Fairall, A. P., Turner, D., Pretorius, M. L., et al. 2004 [arXiv: astro-ph/0411437]

Fioc, M., \& Rocca-Volmerange, B. 1997, A\&A, 326, 950

Fioc, M., \& Rocca-Volmerange, B. 1999 [arXiv: astro-ph/9912179]

Gil de Paz, A., Madore, B. F., \& Pevunova, O. 2003, ApJS, 147, 29

González Delgado, R. M., Leitherer, C., \& Heckman, T. M. 1999, ApJS. 125, 489

Gottlöber, S., Lokas, E., Klypin, A., Hoffman, Y. 2003, MNRAS, 344, 715

Grogin, N. A., \& Geller, M. J. 2000, AJ, 119, 32

Guseva. N. G., Papaderos, P., Izotov, Y. I., et al. 2003, A\&A, 407, 105

Hopp, U., \& Schulte-Ladbeck, R. E. 1995, A\&AS, 111, 527

Hibbard, J. E., van Gorkom, J. H., Rupen, M. P., \& Shiminovich, D., 2001, http://www.cv.nrao.edu/ jhibbard/TSeq

Huchtmeier, W., Hopp, U., \& Kuhn, B. 1997, A\&A, 319, 67

Izotov, Y. I., \& Thuan, T. X. 1999, ApJ, 511, 639

Izotov, Y. I., \& Thuan, T. X. 2004, ApJ, 616, 768

Izotov, Y. I., \& Thuan, T. X. 2005, ApJ, in press

[arXiv:astro-ph/0506498] 
Izotov, Y. I., Lipovetsky, V. A., Chaffee, F. H. et al. 1997a, ApJ, 476, 698

Izotov, Y., Thuan, T. X., \& Lipovetsky, V. 1997b, ApJS, 108, 1

Izotov, Y., Chaffee, F., Foltz, C., et al. 1999, ApJ, 527, 757

Karachentsev, I. G., Karachentseva, V. E., Huchtmeier, W. K., \& Makarov, D. I. 2004, AJ, 127, 2031

Kniazev, A., Pustilnik, S., Masegosa, J. et al. 2000, A\&A, 357, 101

Kniazev, A., Grebel, E., Hao, L., et al. 2003, ApJ, 593, L73

Kniazev, A., Grebel, E., Pustilnik, S., et al. 2004a, AJ, 127, 704

Kniazev A. Y., Pustilnik S. A., Grebel, E., Pramskij A. G., \& Lee, H. 2004b, ApJS, 153, 429

Kunth, D., \& Sargent, W. L. W. 1986, ApJ, 300, 496

Kunth, D., \& Östlin, G. 2000, A\&AR, 10, 1

Larsen, S. S., Brodie, J. P., Elmegreen, B. G. et al. 2001, ApJ, 556, 801

Lee, J. C., Salzer, J. J., \& Melbourne, J. 2004, ApJ, 616, 752

Leitherer, C., Schaerer, D., Goldader, J. D., et al. 1999, ApJS, 123, 3

Lindner, U., Einasto, M., Einasto, J., et al. 1996, A\&A, 314, 1

Lipovetsky, V. A., Chaffee, F. H., Izotov, Y. I., et al. 1999, ApJ, 519, 177

Lu, L., Sargent, W. L. W., Barlow, T., \& Rauch, M. 1998, [arXiv: astro-ph/9802189]

Makarova, L. N., \& Karachentsev, I. D. A\&AS, 1998, 133, 181

Makarova, L. N., Grebel, E., Karachentsev, I. D., et al. 2002, A\&A, 396, 473

Manning, C. 2002, ApJ, 574, 599

Nakos, T., Ofek, E. O., Boumis, P., et al. 2003 A\&A, 402, 1157

Östlin, G., Amram, P., Bergvall, N. et al. 2001, A\&A, 374, 800

Papaderos, P., Izotov, Y. I., Thuan, T. X., et al. 2002, A\&A, 393, 461

Peebles, P. J. E. 2001, ApJ, 557, 495

Plante, S., \& Sauvage, M. 2002, AJ, 124, 1995

Popescu, C. C., Hopp, U., \& Elsaesser, H, 1997, A\&A, 325, 881

Pustilnik S. A., Ugryumov A. V., Lipovetsky V. A., Thuan T. X., \& Guseva, N. G. 1995, ApJ, 443, 499

Pustilnik S. A., Brinks E., Thuan T. X., Lipovetsky V. A., \& Izotov Y. I., 2001, AJ, 121, 1413
Pustilnik, S. A., Martin, J.-M., Huchtmeier, W., et al. 2002, A\&A, 389, 405

Pustilnik, S. A., Kniazev, A. Y., Ugryumov, A. V., \& Pramskij, A. G., 2003a, Ap\&SS, 284, 795

Pustilnik, S. A., Kniazev, A. Y., Pramskij, A. G., Ugryumov, A. V., \& Masegosa, J. 2003b, A\&A, 409, 917

Pustilnik, S. A., Pramskij, A. G., \& Kniazev, A. Y. 2004a, A\&A, 425, 51

Pustilnik, S. A., Kniazev, A. Y., Pramskij, A. G., et al. 2004b, A\&A, 419, 469

Rojas R. R., Vogeley, M. S., Hoyle, F., \& Brinkmann, J. 2004, ApJ, 617,50

Salzer, J. J. 1989, ApJ. 347, 152

Salzer, J. J., Di Serego Alighieri, S., Matteucci, F., Giovanelli, R., \& Haynes, M. 1991, AJ, 101, 1258

Shergin, V. S., Kniazev, A. Y., \& Lipovetsky, V. A. 1996, Astron. Nachr., 2, 95

Schlegel, D., Finkbeiner, D., \& Douglas, M. 1998, ApJ, 500, 525

Searle, L., \& Sargent, W. L. W. 1972, ApJ, 173, 25

Stasińska, G. 1990, A\&AS, 83, 501

Stil, J. M. 1999, Ph.D. Thesis Dwarf Galaxies: Dynamics and Star Formation, Leiden Univ.

Stil, J. M., \& Israel, F. P. 2002a, A\&A, 389, 29

Stil, J. M., \& Israel, F. P. 2002b, A\&A, 389, 42

Thuan T. X., Izotov, Y. I., \& Lipovetsky, V. A. 1997, ApJ, 477, 661

Trentham, N., Möller, O., \& Ramirez-Ruiz, E. 2001, MNRAS, 322, 658

van Dyk, S. D., Puche, D., \& Wong, T. 1998, AJ, 116, 2341

van Zee, L., Haynes, M., Salzer, J. J., \& Broeils, A. 1996, AJ, 112, 129

van Zee, L., Haynes, M., \& Salzer, J. J., 1997, AJ, 114, 2479

van Zee, L., Westpfahl, D., Haynes, M., \& Salzer, J. J. 1998, AJ, 115, 1000

Vazquez, G. A., \& Leitherer, C. 2005, ApJ, 621, 695

Weilbacher, P., \& Fritze-v.Alvensleben, U. 2001, A\&A, 373, L9 